\title{
Supporting Informations:
}

\section{Experimental Section:}

Materials and methods: Acetonitrile, Ethylacetate, Hexane, THF, DMF, Dichloromethane and all other solvents were dried by standard procedure. All the chemicals procured commercially and were purified where necessary. Column chromatography was performed on ACME Silica gel. TLC was performed either on Acme Silica Gel-G coated glass plates and were irradiated with lodine or Kieselgel 60 $\mathrm{GF}_{254}$ coated aluminum plates and were irradiated using UV lamp. 'H NMR spectra were recorded using Varian, Gemini-2000, 200MHz machine, Bruker 400mHz and Bruker WP-80 MHz and Jeol PMX-60 MHz machines in $\mathrm{CDCl}_{3}$ solvent. Chemical shifts are given relative to TMS in ppm $(\delta)$. Multiplicity is indicated using the following abbreviations: s (singlet); d (doublet); dd (doublet of a doublet); ddd (doublet of a doublet of a doublet); $\mathrm{q}$ (quartet) and $\mathrm{m}$ (multiplate). The CI/ EI-mass spectra were conducted on Hewlett Packard, 5989A mass spectrometer and PE SICEX API 300 LC-MS-MS machine using Isobutane gas. Optical rotations were measured in JASCO-DIP 375 polarimeter. FT-IR spectra were recorded on Perkin-Elmer 1650 spectrophotometer using neat sample. Molecular dynamics (MD) calculations for compounds 8c and 9 were carried out using Cerius program on a Silicon graphics Indigo workstation. Charges were calculated using the Charge-equilibration method The CFF9 force field with default parameters were used throughout the simulations. In order to understand the conformational treedom, Simulated Annealing Molecular Dynamics calculations were carried out. The temperature was varied between $300 \mathrm{~K}$ to $1200 \mathrm{~K}$ in the steps of $50 \mathrm{~K}$ for 
(C) 2002 American Chemical Society, J. Org. Chem., Rajesh jo010981d Supporting Info Page 2 100,150 cycles. The molecules were aliowed to equilibrate for $0.5 \mathrm{ps}$ for every change in the temperature. Minimizations were done first with steepest descent, followed by conjugate gradient methods for a maximum of 1000 iterations each or RMS deviation of $0.01 \mathrm{kcal} / \mathrm{mol}$, whichever was earlier. The energy-minimized structures were then subjected to $\mathrm{MD}$ simulations. Various conformers obtained in each $\mathrm{MD}$ run were minimized using the above mentioned minimization protocol.

Methyl-3-(N-phenyl)-3-phenyl-2-methyledine propionate (2a): To a stirred solution of allylacetate 1 a $(234 \mathrm{mg}, 1 \mathrm{mmol}, 1 \mathrm{eq})$ in dry THF $(2 \mathrm{~mL})$ was added $\mathrm{Pd}\left[\left(\mathrm{PPh}_{3}\right)\right]_{4}(0.02$ $\mathrm{mmol}, 0.02 \mathrm{eq}$ ) and aniline ( $93 \mathrm{mg}, 1 \mathrm{mmol}, 1 \mathrm{eq}$ ) and the resulting mixture was stirred at $25^{\circ} \mathrm{C}$ for 2 hours under nitrogen atmosphere. The solvent was removed completely under vacuum and the residue was taken in ethyl acetate and washed with saturated solution of sodium bicarbonate $(3 \times 5 \mathrm{~mL})$ and water $(3 \times 5 \mathrm{~mL})$. Drying (sodium sulfate) and evaporation of the solvent gave a residue, which was chromatographed over silica gel (hexanes) to afford the title compound $2 \mathbf{a}$ as a gum, in $65 \%$ yield. ' $\mathrm{H}$ NMR $(400 \mathrm{MHz}$, $\left.\mathrm{CDCl}_{3}\right) \delta 7.67 \sim 7.18(5 \mathrm{H}, \mathrm{m}) ; 7.11-7.02(2 \mathrm{H}, \mathrm{m}) ; 6.53(1 \mathrm{H}, \mathrm{t}, J=7.6 \mathrm{~Hz}) ; 6.50(2 \mathrm{H}, \mathrm{d}, J=$ $7.6 \mathrm{~Hz}) ; 6.32(1 \mathrm{H}, \mathrm{s}) ; 5.90(1 \mathrm{H}, \mathrm{s}) ; 5.34(1 \mathrm{H}, \mathrm{s}) ; 3.63(3 \mathrm{H}, \mathrm{s}), 1.53(\mathrm{bs}, 1 \mathrm{H}) ; \mathrm{MS}$ (GCMS) m/z: $267\left(\mathrm{M}^{+}\right), 209,191,182,175,116$.

Methyl-3-(N-anisyl)-3-phenyl-2-methyledine propionate (2b): To a stirred solution of allylacetate 1 a $\left(234 \mathrm{mg}, 1 \mathrm{mmol}\right.$, leq) in dry THF $(2 \mathrm{~mL})$ was added $\mathrm{Pd}\left[\left(\mathrm{PPh}_{3}\right)\right]_{4}(0.02$ mmol, $0.02 \mathrm{eq})$ and $\mathrm{p}$-anisidine $(123 \mathrm{mg}, 1 \mathrm{mmol}, 1 \mathrm{eq})$ and the resulting mixture was stirred at $25^{\circ} \mathrm{C}$ for 2 hours under $\mathrm{N}_{2}$ atmosphere. Rest of the procedure is same as $2 \mathbf{a}$. Gum, yield $=59 \% ;{ }^{1} \mathrm{H}$ NMR $\left(400 \mathrm{MHz} \mathrm{CDCl}_{3}\right) \delta 7.31 \sim 7.18(5 \mathrm{H}, \mathrm{m}) ; 6.69(2 \mathrm{H}, \mathrm{d}, J=$ $9.04 \mathrm{~Hz}) ; 6.47(2 \mathrm{H}, \mathrm{d}, J=8.8 \mathrm{~Hz}) ; 6.33(1 \mathrm{H}, \mathrm{s}) ; 5.89(1 \mathrm{H}, \mathrm{d}, J=1.20 \mathrm{~Hz}) ; 5.26(1 \mathrm{H}, \mathrm{s})$ 
(C) 2002 American Chemical Society, J. Org. Chem., Rajesh jo010981d Supporting Info Page 3 ; 3.66 (3H. s); $3.69(3 \mathrm{H}, \mathrm{s}) ; \mathrm{MS}(\mathrm{GCMS}) \mathrm{m} / \mathrm{z}: 297\left(\mathrm{M}^{+}\right), 212,122(100 \%), 115$; FTIR (neat) : $3385,2925,1719,1596,1512,1243 \mathrm{~cm}^{-1}$

Methyl-3-(N-toluidyl)-3-phenyl-2-methyledine propionate (2c): To a stirred solution of allylacetate 1 a $(234 \mathrm{mg}, 1 \mathrm{mmol}$. $1 \mathrm{eq})$ in dry THF $(2 \mathrm{~mL})$ was added $\mathrm{Pd}\left[\left(\mathrm{PPh}_{3}\right)\right]_{4}$ (0.02 mmol, $0.02 \mathrm{eq})$ and p-toluidine (107 $\mathrm{mg}, 1 \mathrm{mmol}, 1 \mathrm{eq})$ and the resulting mixture was stirred at $25^{\circ} \mathrm{C}$ for 2 hours under $\mathrm{N}_{2}$ atmosphere. Rest of the procedure is same as $2 \mathbf{a}$. Gum, yield $=61 \% ;{ }^{1} \mathrm{H}$ NMR $\left(400 \mathrm{MHz} \mathrm{CDCl}_{3}\right) \delta 7.40 \sim 7.08(5 \mathrm{H}, \mathrm{m}) ; 6.99(2 \mathrm{H}, \mathrm{d}, J=7$ $\mathrm{Hz}) ; 6.40(2 \mathrm{H}, \mathrm{d}, J=6.6 \mathrm{~Hz}) ; 6.30(1 \mathrm{H}, \mathrm{s}) ; 5.88(1 \mathrm{H}, \mathrm{d}, J=1.24 \mathrm{~Hz}) ; 5.30(1 \mathrm{H}, \mathrm{s}) ; 3.62$ $(3 \mathrm{H}, \mathrm{s}) ; 2.15(3 \mathrm{H}, \mathrm{s}) ; \mathrm{MS}(\mathrm{GCMS}) \mathrm{m} / \mathrm{z}: 281\left(\mathrm{M}^{+}\right), 191,175,91$.

Methyl-3-(N-4-chlorophenyl) amino-3-phenyl-2-methyledine propionate (2d): To a stirred solution of allylacetate 1a $(234 \mathrm{mg}, 1 \mathrm{mmol}, \mathrm{l} \mathrm{eq})$ in dry THF (2 mL) was added $\operatorname{Pd}\left[\left(\mathrm{PPh}_{3}\right)\right]_{4}(0.02 \mathrm{mmol}, 0.02 \mathrm{eq})$ and p-chloroaniline $(127.57 \mathrm{mg}, 1 \mathrm{mmol}, 1 \mathrm{eq})$ and the resulting mixture was stirred at $25^{\circ} \mathrm{C}$ for 2 hours under $\mathrm{N}_{2}$ atmosphere. Rest of the procedure is similar to $2 \mathrm{a}$. yield: $56 \%,{ }^{\prime} \mathrm{H} \mathrm{NMR}\left(400 \mathrm{MHz}, \mathrm{CDCl}_{3}\right) \delta 7.29 \sim 7.22(5 \mathrm{H}, \mathrm{m})$ ; $7.02(2 \mathrm{H}, \mathrm{d}, J=2.4 \mathrm{~Hz}) ; 6.42(2 \mathrm{H}, \mathrm{d}, J=8.4 \mathrm{~Hz}) ; 6.32(1 \mathrm{H}, \mathrm{s}) ; 5.84(1 \mathrm{H}, \mathrm{s}) ; 5.26$ $(\mathrm{lH}, \mathrm{s}) ; 4.03(\mathrm{bs}, 1 \mathrm{H}) ; 3.64(3 \mathrm{H}, \mathrm{s}) ; \mathrm{MS}(\mathrm{GCMS}) \mathrm{m} / \mathrm{z}: 301\left(\mathrm{M}^{+}\right), 218,116,115$ (base peak), 91; FTIR (neat) : 3395, 2925, 1720, 1600,1497, $1439 \mathrm{~cm}^{-1}$

Methyl-3-(N-anisyl)-3-(4-methoxy-phenyl)-2-methyledine propionate (2e): To a stirred solution of allylacetate $\mathbf{l b}$ (26 $4 \mathrm{mg} .1 \mathrm{mmol}, 1 \mathrm{eq}$ ) in dry THF (2 mL) was added $\mathrm{Pd}\left[\left(\mathrm{PPh}_{3}\right)\right]_{4}(0.02 \mathrm{mmol}, 0.02 \mathrm{eq})$ and p-anisidine $(123 \mathrm{mg}, 1 \mathrm{mmol}, 1 \mathrm{eq})$ and the resulting mixture was stirred at $25^{\circ} \mathrm{C}$ for 2 hours under $\mathrm{N}_{2}$ atmosphere. Rest of the procedure is same as 2a. Gum. ${ }^{\mathrm{I}} \mathrm{H}$ NMR $\left(400 \mathrm{MHz}, \mathrm{CDCl}_{3}\right) \delta 7.23(2 \mathrm{H}, J=8.5 \mathrm{~Hz}) ; 6.79$ 
(C) 2002 American Chemical Society, J. Org. Chem., Rajesh jo010981d Supporting Info Page 4 $(2 \mathrm{H}, \mathrm{d}, J=6.6 \mathrm{~Hz}) ; 6.69(2 \mathrm{H}, \mathrm{d}, J=7.32 \mathrm{~Hz}) ; 6.5(2 \mathrm{H}, \mathrm{d}, J=8.8 \mathrm{~Hz}) ; \dot{6} .29(1 \mathrm{H}, \mathrm{s})$; $5.88(1 \mathrm{H}, \mathrm{s}) ; 5.29(1 \mathrm{H}, \mathrm{s}) ; 3.62(3 \mathrm{H}, \mathrm{s}), 3.61(3 \mathrm{H}, \mathrm{s}), 3.60(3 \mathrm{H}, \mathrm{s}), 2.03(\mathrm{bs}, 1 \mathrm{H})$.

Methyl-3-(N-anisyl)-3-(4-chlorophenyl)-2-methyledine propionate (2f): To a stirred solution of allylacetate $1 \mathrm{c}(252 \mathrm{mg}, 1 \mathrm{mmol}, 1 \mathrm{eq})$ in dry THF $(2 \mathrm{~mL})$ was added $\mathrm{Pd}\left[\left(\mathrm{PPh}_{3}\right)\right]_{4}(0.02 \mathrm{mmol}, 0.02 \mathrm{eq})$ and $\mathrm{p}$-anisidine (i23 $\mathrm{mg}, 1 \mathrm{mmol}$, leq) and the resulting mixture was stirred at $25^{\circ} \mathrm{C}$ for 2 hours under $\mathrm{N}_{2}$ atmosphere. Rest of the procedure is same as $2 \mathbf{2 a}$. Gum. ' $\mathrm{H}$ NMR $\left(400 \mathrm{MHz}, \mathrm{CDCl}_{3}\right) \delta$ 7.37-7.26 $(4 \mathrm{H}, \mathrm{m}) ; 6.75$ $(2 \mathrm{H}, \mathrm{d}, J=2.4 \mathrm{~Hz}) ; 6.53(2 \mathrm{H}, \mathrm{d}, J=2.2 \mathrm{~Hz}) ; 6.40(1 \mathrm{H}, \mathrm{s}) ; 5.92(1 \mathrm{H}, \mathrm{s}) ; 5.30(1 \mathrm{H}, \mathrm{s}) ; 3.72$ $(3 \mathrm{H}, \mathrm{s}) ; 3.70(3 \mathrm{H}, \mathrm{s})$.

Methyl-3-(N-benzyl)-3-(4-methoxy-phenyl)-2-methylidine propionate (2g): To a stirred solution of allylacetate $\mathbf{1 b}(264 \mathrm{mg}, 1 \mathrm{mmol}, 1 \mathrm{eq})$ in dry THF $(2 \mathrm{~mL})$ was added $\operatorname{Pd}\left[\left(\mathrm{PPh}_{3}\right)\right]_{4}(0.02 \mathrm{mmol}, 0.02 \mathrm{eq})$ and benzylamine $(107 \mathrm{mg}, 1 \mathrm{mmol}, 1 \mathrm{eq})$ and the resulting mixture was stirred at $25^{\circ} \mathrm{C}$ for 2 hours under $\mathrm{N}_{2}$ atmosphere. Rest of the procedure is same as 2a. Gum. ' $\mathrm{H}$ NMR $\left(80 \mathrm{MHz}, \mathrm{CDCl}_{3}\right) \delta 7.81(2 \mathrm{H}, \mathrm{m}) ; 7.62-7.25(\mathrm{~m}$, 4H); $6.93(\mathrm{~m}, 2 \mathrm{H}) ; 6.37(1 \mathrm{H}, \mathrm{s}) ; 6.03(1 \mathrm{H}, \mathrm{s}) ; 5.06(1 \mathrm{H}, \mathrm{s}) ; 3.78(3 \mathrm{H}, \mathrm{s}) ; 3.75(3 \mathrm{H}, \mathrm{s})$; $3.62(\mathrm{~m}, 2 \mathrm{H}) ; 2.59(\mathrm{bs}, 1 \mathrm{H})$.

Methyl-(N-phenyl)-3-amino-2-benzylidene propionate (3a): To a stirred solution of allylacetate 1a $(1 \mathrm{mmol}, 234 \mathrm{mg}, 1 \mathrm{eq})$ in acetonitrile $(3 \mathrm{~mL})$ was added $\mathrm{Pd}\left[\left(\mathrm{PPh}_{3}\right)\right]_{4}$ ( $0.02 \mathrm{mmol}, 0.02 \mathrm{eq}$ ) followed by aniline ( $1 \mathrm{mmol}, 0.093 \mathrm{gm} .1 \mathrm{eq}$ ) under $\mathrm{N}_{2}$ and refluxed at $80^{\circ} \mathrm{C}$ for 2 hours. After that solvent was removed in vaccum, residue was taken in ethyl acetate, washed with sodium bicarbonate solution $(3 \times 10 \mathrm{~mL})$. The combined organic layer was dried over anhydrous sodium sulfate, Concentration resulted in a thick gummy residue which was chromatographed (hexanes) yielding $\mathbf{3 a}$ in $68 \%$ yield. 'H NMR (80 
(C) 2002 American Chemical Society, J. Org. Chem., Rajesh jo010981d Supporting Info Page 5 $\left.\mathrm{MHz}_{\mathrm{CDCl}}\right) \delta 8.12(1 \mathrm{H}, \mathrm{s}) ; 7.5 \sim 7.15(\mathrm{iOH}, \mathrm{m}) ; 6.8(1 \mathrm{H}, \mathrm{m}) ; 3.87(3 \mathrm{H}, \mathrm{s}) ; 3.65(2 \mathrm{H}$, $\mathrm{m})$.

Methyl-(N-anisyl)-3-amino-2-benzylidene propionate (3b): To a stirred solution of allylacetate 1 a $(1 \mathrm{mmol}, 234 \mathrm{mg}, 1 \mathrm{eq})$ in acetonitrile $(3 \mathrm{~mL})$ was added $\mathrm{Pd}\left[\left(\mathrm{PPh}_{3}\right)\right]_{4}$ (0.02 mmol, $0.02 \mathrm{eq}$ ) followed by p-anisidine ( $1 \mathrm{mmol} .0 .123 \mathrm{gm}, 1 \mathrm{eq}$ ) under $\mathrm{N}_{2}$ and refluxed at $80^{\circ} \mathrm{C}$ for 2 hours. Rest of the procedure is same like 3a, gum, Yield $=61 \% ;{ }^{1} \mathrm{H}$ NMR $\left(80 \mathrm{MHz}, \mathrm{CDCl}_{3}\right) \delta 8.06(1 \mathrm{H}, \mathrm{s}) ; 7.56 \sim 7.25(7 \mathrm{H}, \mathrm{m}) ; 6.65-6.43(2 \mathrm{H}, \mathrm{m}) ; 5.53$ $(1 \mathrm{H}, \mathrm{m}) ; 3.78(2 \mathrm{H}, \mathrm{m}) ; 3.62(6 \mathrm{H}, \mathrm{s})$.

Methyl-(N-toludyl)-3-amino-2-benzylidene propionate (3c): To a stirred solution of allylacetate 1a (1 mmol, $234 \mathrm{mg}, 1 \mathrm{eq})$ in acetonitrile $(3 \mathrm{~mL})$ was added $\mathrm{Pd}\left[\left(\mathrm{PPh}_{3}\right)\right]_{4}$ (0.02 mmol, $0.02 \mathrm{eq})$ followed by p-toluidine ( $1 \mathrm{~mol}, 0.107 \mathrm{gm}, 1 \mathrm{eq})$ under $\mathrm{N}_{2}$ and refluxed at $80^{\circ} \mathrm{C}$ for 2 hours. Rest of the procedure is same like $3 \mathbf{a}$, gum, Yield $=57 \%$; ${ }^{1} \mathrm{H}$ NMR $\left(80 \mathrm{MHz}_{,} \mathrm{CDCl}_{3}\right) \delta 7.9(1 \mathrm{H}, \mathrm{s}) ; 7.48 \sim 7.19(5 \mathrm{H}, \mathrm{m}) ; 7.09-6.96(2 \mathrm{H}, \mathrm{m}) ; 6.64-6.58$ $(2 \mathrm{H}, \mathrm{m}) ; 5.38(\mathrm{~m}, 1 \mathrm{H}), 3.67(3 \mathrm{H}, \mathrm{s}) ; 3.54(2 \mathrm{H}, \mathrm{m}), 2.19(3 \mathrm{H}, \mathrm{s})$.

Methyl-(N-4-chlorophenyl)-3-amino-2-benzylidene propionate (3d): To a stirred solution of allylacetate $1 \mathrm{a}(1 \mathrm{mmol}, 234 \mathrm{mg}, 1 \mathrm{eq})$ in acetonitrile ( $3 \mathrm{~mL}$ ) was added $\mathrm{Pd}\left[\left(\mathrm{PPh}_{3}\right)\right]_{4}(0.02 \mathrm{mmol}, 0.02 \mathrm{eq})$ followed by p-chloroaniline (I mmol, $\left.0.123 \mathrm{gm}, 1 \mathrm{eq}\right)$ under $\mathrm{N}_{2}$ and refluxed at $80^{\circ} \mathrm{C}$ for 2 hours. Rest of the procedure is same like $3 \mathrm{~d}$, gum, Yield $=60 \%$ : 'H NMR $\left(80 \mathrm{MHz}_{\mathrm{C}} \mathrm{CDCl}_{3}\right) \delta 7.93(1 \mathrm{H} . \mathrm{s}) ; 7.51 \sim 7.29(5 \mathrm{H}, \mathrm{m}) ; 6.74-6.58$ $(2 \mathrm{H}, \mathrm{m}) ; 5.93(1 \mathrm{H}, \mathrm{m}) ; 3.83(3 \mathrm{H}, \mathrm{s}) ; 3.77(2 \mathrm{H}, \mathrm{m})$.

Methyl-(N-4-methoxyphenyl)-3-amino-2-(4-methoxyphenyl)-methylidine propionate (3e): To a stirred solution of allylacetate 1 b $(264 \mathrm{mg}, 1 \mathrm{mmol}, \mathrm{leq})$ in dry THF $(2 \mathrm{~mL})$ was added $\mathrm{Pd}\left[\left(\mathrm{PPh}_{3}\right)\right]_{4}(0.02 \mathrm{mmol}, 0.02 \mathrm{eq})$ and p-anisidine $(123 \mathrm{mg}, 1 \mathrm{mmol}, \mathrm{leq})$ and 
(C) 2002 American Chemical Society, J. Org. Chem., Rajesh jo010981d Supporting Info Page 6 the resulting mixture was stirred at $25^{\circ} \mathrm{C}$ for 2 hours under $\mathrm{N}_{2}$ atmosphere. Rest of the procedure is same as 3e. Gum, yield $=76 \% ;{ }^{1} \mathrm{H} \mathrm{NMR}\left(80 \mathrm{MHz}, \mathrm{CDCl}_{3}\right) \delta 8.31(\mathrm{~s}, 1 \mathrm{H})$; $7.87(\mathrm{~m}, 2 \mathrm{H}) ; 7.56-7.25(\mathrm{~m}, 4 \mathrm{H}) ; 6.93(\mathrm{~m}, 2 \mathrm{H}) ; 3.81(\mathrm{~s}, 2 \mathrm{H}), 3.65(\mathrm{~s}, 3 \mathrm{H}), 3.62(\mathrm{~s}, 3 \mathrm{H})$; $2.62(\mathrm{bs}, 1 \mathrm{H})$.

Methyl-(N-4-methoxyphenyl)-3-amino-2-(4-chlorophenyl)-methylidine propionate (3f): To a stirred solution of allylacetate $1 \mathrm{c}(252 \mathrm{mg}, 1 \mathrm{mmol}, \mathrm{leq})$ in dry THF (2mL) was added $\mathrm{Pd}\left[\left(\mathrm{PPh}_{3}\right)\right]_{4}(0.02 \mathrm{mmol}, 0.02 \mathrm{eq})$ and p-anisidine (123mg, Immol, leq) and the resulting mixture was stirred at $25^{\circ} \mathrm{C}$ for 2 hours under $\mathrm{N}_{2}$ atmosphere. Rest of the procedure is same as 3f. Gum, yield=68\%; ' $\mathrm{H}$ NMR $\left(80 \mathrm{MHz}, \mathrm{CDCl}_{3}\right) \delta 7.87(\mathrm{~s}, 1 \mathrm{H})$; $7.34(\mathrm{~m}, 2 \mathrm{H}) ; 7.21(\mathrm{~m}, 2 \mathrm{H}) ; 7.12(\mathrm{~m}, 2 \mathrm{H}) ; 6.75(\mathrm{~m}, 2 \mathrm{H}) ; 3.84(\mathrm{~s}, 2 \mathrm{H}), 3.78(\mathrm{~s}, 6 \mathrm{H})$.

Methyl-(N-benzyl)-3-amino-2-(4-methoxyphenyl)-methylidine propionate (3g): To a stirred solution of allylacetate $\mathbf{1 b}(264 \mathrm{mg}, 1 \mathrm{mmol}, 1 \mathrm{eq})$ in dry THF (2 mL) was added $\mathrm{Pd}\left[\left(\mathrm{PPh}_{3}\right)\right]_{4}(0.02 \mathrm{mmol}, 0.02 \mathrm{eq})$ and benzylamine $(107 \mathrm{mg}, 1 \mathrm{mmol}, 1 \mathrm{eq})$ and the resulting mixture was stirred at $25^{\circ} \mathrm{C}$ for 2 hours under $\mathrm{N}_{2}$ atmosphere. Rest of the procedure is same as 3g. Gum, yield $=66 \%$; ${ }^{1} \mathrm{H}$ NMR $\left(80 \mathrm{MHz}, \mathrm{CDCl}_{3} \delta 8.31(\mathrm{~s}, 1 \mathrm{H})\right.$; $7.75(\mathrm{~m}, 2 \mathrm{H}) ; 7.53(\mathrm{~m}, 5 \mathrm{H}) ; 7.0(\mathrm{~m}, 2 \mathrm{H}) ; 3.84(\mathrm{~s}, 3 \mathrm{H}), 3.78(\mathrm{~m}, 5 \mathrm{H}), 3.62(\mathrm{~m}, 2 \mathrm{H}) ; 2.60$ (bs, $1 \mathrm{H}$ ).

Methyl-(N-benzyl)-3-amino-2-(4-chlorophenyl)-methylidine propionate (3h): To a stirred solution of allylacetate 1 c $(252 \mathrm{mg} .1 \mathrm{mmol}, 1 \mathrm{eq})$ in dry THF (2 mL) was added $\mathrm{Pd}\left[\left(\mathrm{PPh}_{3}\right)\right]_{4}(0.02 \mathrm{mmol}, 0.02 \mathrm{eq})$ and benzylamine $(107 \mathrm{mg}, 1 \mathrm{mmol}, 1 \mathrm{eq})$ and the resulting mixture was stirred at $25^{\circ} \mathrm{C}$ for 2 hours under $\mathrm{N}_{2}$ atmosphere. Rest of the procedure is same as $3 \mathbf{h}$. Gum, yield $=78 \% ;{ }^{1} \mathrm{H}$ NMR $\left(80 \mathrm{MHz}, \mathrm{CDCl}_{3}\right) \delta 7.78(\mathrm{~s}, 1 \mathrm{H})$; 
(C) 2002 American Chemical Society, J. Org. Chem., Rajesh jo010981d Supporting Info Page 7 $7.43(\mathrm{~m} .2 \mathrm{H}) ; 7.31(\mathrm{~m}, 5 \mathrm{H}) ; 6.98(\mathrm{~m}, 2 \mathrm{H}) ; 3.84(\mathrm{~s}, 3 \mathrm{H}) ; 3.75(\mathrm{~s} .2 \mathrm{H}) ; 3.68(\mathrm{~m}, 2 \mathrm{H}) ;$ $2.25($ bs, $1 \mathrm{H})$.

Methyl-3-|methyl-(L)-4-hydroxyprolinate|-2-benzylidene propionate (4a): To a stirred solution of allylacetate 1a $(567 \mathrm{mg}, 2.42 \mathrm{mmol}$, leq) in $6 \mathrm{~mL}$ acetonitrile was treated with Methyl-(L)-Prolinate hydrochloride (482mg. 2.904mmol, 1.2ey), triethylamine $(0.812 \mathrm{~mL}, 5.815 \mathrm{mmol}, 2.4 \mathrm{eq})$ and $\mathrm{Pd}\left[\left(\mathrm{PPh}_{3}\right)\right]_{4}(0.0484 \mathrm{mmol}, 0.058 \mathrm{gm}$, $0.02 \mathrm{eq})$ under Argon and was stirred for 2 hours. After that solvent was removed in vacuum, It was taken in ethyl acetate, washed with saturated sodium bicarbonate solution $(3 \times 10 \mathrm{~mL})$ and water. The combined organic layers were dried over sodium Sulfate, concentration and evaporation resulted in a thick gummy residue which were chromatographed (hexanes) yielding $3 \mathbf{b}$ as a Liquid in $67 \%$ yiejd. 'H NMR $(60 \mathrm{MHz}$, $\left.\mathrm{CDCl}_{3}\right) \delta 7.9(1 \mathrm{H}, \mathrm{s}) ; 7.8 \sim 7.6(2 \mathrm{H}, \mathrm{m}) ; 7.4 \sim 7.1(\mathrm{~m}, 3 \mathrm{H}) ; 3.9(3 \mathrm{H}, \mathrm{s}) ; 3.7(3 \mathrm{H}, \mathrm{s}) ; 3.6$ $(1 \mathrm{H}, \mathrm{d}, J=3 \mathrm{~Hz}) ; 3.4(1 \mathrm{H}, \mathrm{d}, J=3 \mathrm{~Hz}) ; 3.3\left(1 \mathrm{H},{ }^{\mathrm{t}}, J=8 \mathrm{~Hz}\right) ; 3.00(1 \mathrm{H}, \mathrm{m}) ; 2.55$ $(1 \mathrm{H}, \mathrm{dd}, J=8 \mathrm{~Hz}, 16 \mathrm{~Hz}) ; 2.2 \sim 1.5(4 \mathrm{H}, \mathrm{m}) ; \quad \mathrm{IR}\left(\mathrm{CCl}_{4}\right): 3060.3020,2960,1730$, $1710,1700,1480 \mathrm{~cm}^{-1},[\alpha]_{\mathrm{D}}=-25.61,(\mathrm{c}=0.009, \mathrm{MeOH})$.

Methyl-3-|methyl-(L)-4-hydroxyprolinate]-3-butyl-2-methylidene propionate (E4b): To a stirred solution of allylacetate $1 \mathrm{~d}(640 \mathrm{mg}, 3 \mathrm{mmol}$. $1 \mathrm{eq})$ in $6 \mathrm{~mL}$ acetonitrile was treated with Methyl-(L)-Prolinate hydrochloride (652 mg. $3.6 \mathrm{mmol}, 1.2 \mathrm{eq}$ ), triethylamine (727 mg, $7.2 \mathrm{mmol}, 2.4 \mathrm{eq})$ and $\mathrm{Pd}\left[\left(\mathrm{PPh}_{3}\right)\right]_{4}(0.069 \mathrm{mmol}, 0.06 \mathrm{gm}, 0.02$ eq) under Argon and was refluxed for 2 hours. Rest of the procedure is same like $4 \mathbf{a}$, Liquid. Yield $=72 \%$; ${ }^{1} \mathrm{H}$ NMR $\left.(400) \mathrm{MHz}, \mathrm{CDCl}_{3}\right) \delta 7.28(1 \mathrm{H}$. bs $) ; 6.94(1 \mathrm{H}, \mathrm{t}, J=7.56$ $\mathrm{Hz}) ; 4.44-4.39(\mathrm{~m}, 1 \mathrm{H}) ; 3.74(3 \mathrm{H}, \mathrm{s}) ; 3.71(3 \mathrm{H}, \mathrm{s}) ; 3.60(2 \mathrm{H}, \mathrm{d}, J=2.88 \mathrm{~Hz}) ; 3.37(1 \mathrm{H}, \mathrm{dd}$, $J=5.6 \mathrm{~Hz} .10 \mathrm{~Hz}) ; 2.57(1 \mathrm{H}, \mathrm{dd} . J=3.6 \mathrm{~Hz}, 10 \mathrm{~Hz}) ; 2.32(1 \mathrm{H}, \mathrm{m}) ; 2.08(1 \mathrm{H}, \mathrm{ddd}, J=$ 
(C) 2002 American Chemical Society, J. Org. Chem., Rajesh jo010981d Supporting Info Page 8 $3.56 \mathrm{~Hz}, 8.04 \mathrm{~Hz} .13 .36 \mathrm{~Hz}) ; 2.19(2 \mathrm{H}, \mathrm{t}, J=7.321 \mathrm{lz}) .1 .8-1.67(1 \mathrm{H}, \mathrm{m}), 0.93(\mathrm{~d}, 3 \mathrm{H}, J$ $=6.6 \mathrm{~Hz}), 0.92(\mathrm{~d} .3 \mathrm{H}, J=6.6 \mathrm{~Hz}): \quad \mathrm{IR}\left(\mathrm{CCl}_{4}\right): 3600-3020(\mathrm{br}), 2940,2860,1730,1710$, 1710. $1630 \mathrm{~cm}^{-1} ;[\alpha]_{\mathrm{D}}=-27.33\left(\mathrm{c}=0.015, \mathrm{CH}_{2} \mathrm{Cl}_{2}\right)$.

Methyl-3-(methyl-L-4-hydroxyprolinate)-2-(4-methoxybenzylidene)-propionate (4c): To a stirred solution of allyl acetate $1 \mathrm{~b}(792 \mathrm{mg}, 3 \mathrm{mmol}, 1 \mathrm{eq})$ in $6 \mathrm{~mL}$ acetonitrile was treated with Methyl-(L)-4-hydroxy-Prolinate hydrochloride $(652 \mathrm{mg}, 3.6 \mathrm{mmol}, 1.2$ eq), triethylamine(1.003 mL, $7.2 \mathrm{mmol}, 2.4 \mathrm{eq})$ and $\mathrm{Pd}\left[\left(\mathrm{PPh}_{3}\right)\right]_{4}(0.09 \mathrm{mmol}, 0.1 \mathrm{gm}$, $0.03 \mathrm{eq}$ ) and was refluxed under Argon for 2 hours. Rest of the procedure is same like $\mathbf{4 a}$, Liquid, Yield $=59 \%$; ' $\mathrm{H}$ NMR $\left(400 \mathrm{MHz}^{\left.-\mathrm{CDCl}_{3}\right)} \delta 7.8(1 \mathrm{H} . \mathrm{s}) ; 7.63(2 \mathrm{H}, \mathrm{d}, J=8.8\right.$ $\mathrm{Hz}) ; 4.42(1 \mathrm{H}, \mathrm{m}) ; 3.84(3 \mathrm{H}, \mathrm{s}) ; 3.81(3 \mathrm{H}, \mathrm{s}) ; 3.74(2 \mathrm{H}, \mathrm{d}, J=6.12 \mathrm{~Hz}) ; 3.65(\mathrm{~s}, 3 \mathrm{H})$; $3.31(1 \mathrm{H}, \mathrm{dd}, J=5.4 \mathrm{~Hz}, 10.28 \mathrm{~Hz}) ; 2.63(1 \mathrm{H}, \mathrm{dd}, J=3.6 \mathrm{~Hz}, 10 \mathrm{~Hz}) ; 2.22(1 \mathrm{H}, \mathrm{m})$; 2.15 2.04 (2H,m); Mass (EI) : $350\left(\mathrm{M}^{+}\right)(3.7 \%), 337,291(45.3 \%), 206(100 \%), 146$ $(96.5 \%) ;$ IR $\left(\mathrm{CCl}_{4}\right): 3700 \sim 3100,2830,1725,1710,1590,1495 \mathrm{~cm}^{-1} ;[\alpha]_{\mathrm{D}}^{25}=-53.33(\mathrm{c}=$ $\left.0.007, \mathrm{CH}_{2} \mathrm{Cl}_{2}\right)$.

Methyl-3-|methyl-(L)-4-hydroxyprolinate]-3-propyl-2-methylidene propionate (4d): To a stirred solution of allylacetate $1 \mathrm{e}(\mathrm{gg}, 5 \mathrm{mmol}, 1 \mathrm{eq})$ in $10 \mathrm{~mL}$ acetonitrile was treated with Methyl-(L)-Prolinate hydrochloride (1.089g, $6 \mathrm{mmol}, 1.2 \mathrm{eq})$, triethylamine $(1.21 \mathrm{mg}$, $12 \mathrm{mmol}, 2.4 \mathrm{eq})$ and $\mathrm{Pd}\left[\left(\mathrm{PPh}_{3}\right)\right]_{4}(0.173 \mathrm{mmol}, 0.15 \mathrm{gm}, 0.034 \mathrm{eq})$ under Argon and was refluxed for 2 hours. Rest of the procedure is same like 4 a. Liquid, Yield $=61 \%$; ${ }^{\prime} \mathrm{H}$ NMR $\left(60 \mathrm{MHz} . \mathrm{CDCl}_{3}\right) \delta 7.9(1 \mathrm{H}$. bs $) ; 6.7(1 \mathrm{H}, \mathrm{d}, y=11 \mathrm{~Hz}) ; 4.5(\mathrm{~m}, 1 \mathrm{H}) ; 3.9(3 \mathrm{H}, \mathrm{s}) ;$ $3.8(3 \mathrm{H}, \mathrm{s}) ; 3.70(1 \mathrm{H}, \mathrm{s}) ; 3.5(1 \mathrm{H.s}) ; 3.3(1 \mathrm{H}, \mathrm{m}) ; 2.9(2 \mathrm{H}, \mathrm{s}) ; 2.6(1 \mathrm{H}, \mathrm{dd}, J=4$ Hz. $10 \mathrm{~Hz}) ; 2.3(2 \mathrm{H}$. dd. $J=2.3 \mathrm{~Hz} .10 \mathrm{~Hz}) .2 .2(1 \mathrm{H.} m) .1 .1(\mathrm{~d} .3 \mathrm{H} . J=8 \mathrm{~Hz}, 6 \mathrm{~Hz})$; 
(C) 2002 American Chemical Society, J. Org. Chem., Rajesh jo010981d Supporting Info Page 9 IR $\left(\mathrm{CCl}_{4}\right): 3600-3020(\mathrm{br}), 3040,2940,1730,1690,1610,1440 \mathrm{~cm}^{-1} ;[\alpha]_{1)}^{-}=-44.6(\mathrm{c}=$ ().009. $\left.\mathrm{CH}_{2} \mathrm{Cl}_{2}\right)$.

Methyl-3-|methyl-(L)-4-hydroxyprolinate|-3-propyl-2-methylidene propionate (4e): To a stirred solution of allylacetate $1 \mathrm{f}(520 \mathrm{mg}, 2 \mathrm{mmol}, 1 \mathrm{eq})$ in $4 \mathrm{~mL}$ acetonitrile was treated with Methyl-(L)-Prolinate hydrochloride (435 mg, $2.4 \mathrm{mmol}, 1.2 \mathrm{eq}$ ), triethylamine (1484 mg, $4.8 \mathrm{mmol}, 2.4 \mathrm{eq})$ and $\mathrm{Pd}\left[\left(\mathrm{PPh}_{3}\right)\right]_{4}(0.04 \mathrm{mmol}, 0.046 \mathrm{gm}, 0.02$ eq) under Argon and was refluxed for 2 hours. Rest of the procedure is same like 4a. Liquid. Yield $=57 \%$; ' $\mathrm{H}$ NMR $\left(60 \mathrm{MHz}, \mathrm{CDCl}_{3}\right) \delta 7.7(1 \mathrm{H}, \mathrm{d}, J=12 \mathrm{~Hz}) ; 7.2-7.0(\mathrm{~m}$, $3 \mathrm{H}) .6 .8(1 \mathrm{H}, \mathrm{d} . J=16 \mathrm{~Hz}) ; 4.3(\mathrm{~m}, 1 \mathrm{H}) ; 3.8(3 \mathrm{H}, \mathrm{s}) ; 3.7(3 \mathrm{H}, \mathrm{s}) ; 3.60(2 \mathrm{H}, \mathrm{s}) ; 3.3(1 \mathrm{H}$, m): $2.6(1 \mathrm{H}, \mathrm{dd}, J=4 \mathrm{~Hz}, 11 \mathrm{~Hz}) ; 2.1(2 \mathrm{H}, \mathrm{m}) ; \mathrm{IR} \quad\left(\mathrm{CCl}_{4}\right): 3600-3100(\mathrm{br}), 3040$, $2940,1730,1690,1610,1440 \mathrm{~cm}^{-1} ;[\alpha]_{\mathrm{D}}=-21.48\left(\mathrm{c}=0.002,\left(\mathrm{CH}_{2} \mathrm{Cl}_{2}\right)\right.$.

Methyl-3-|methyl-(L)-serinate]- 2-benzylidene propionate (4f): To a stirred solution of allylacetate 1 a $(567 \mathrm{mg}, 2.42 \mathrm{mmol}, 1 \mathrm{eq})$ in $10 \mathrm{~mL}$ acelonitrile was treated with Methyl-(L)-Serinate hydrochloride (312 mg, $2.92 \mathrm{mmol}, 1.2 \mathrm{eq})$, tricthylamine $(0.812 \mathrm{~mL}$ $.5 .815 \mathrm{mmol} .2 .4 \mathrm{eq})$ and $\mathrm{Pd}\left[\left(\mathrm{PPh}_{3}\right)\right]_{4}(0.0484 \mathrm{mmol}, 0.058 \mathrm{gm}, 0.02 \mathrm{eq})$ under Argon and was refluxed for 2 hours Rest of the procedure is same like 4a. Liquid, Yield $=66 \%$; 'H NMR (400 MH\%, $\left.\mathrm{CDCl}_{3}\right) \delta 7.82(1 \mathrm{H}, \mathrm{s}), 7.42-7.26(6 \mathrm{H} . \mathrm{m}) ; 3.85(3 \mathrm{H} . \mathrm{s}) ; 3.79(2 \mathrm{H}$, $\mathrm{dd}, J=5.12 .3 .64 \mathrm{~Hz}) ; 3.74(3 \mathrm{H}, \mathrm{s}) ; 3.68(2 \mathrm{H}, \mathrm{s}) ; 3.4-3.37(1 \mathrm{H}, \mathrm{m}) ; 2.15(1 \mathrm{H}, \mathrm{bs})$.

\section{Coupling of (L)-leucine methyl ester with N-Cinnamoylated-dehydro-aminoacid} (6a): To a stirred solution of the dehydro amine $3 \mathrm{~b}(1.841 \mathrm{~g}, 6.2 \mathrm{mmol}, 1 \mathrm{eq})$ in $6.2 \mathrm{~mL}$ dry DCM was treated with trans-cinnamoyl chloride (1.033 gm, $6.2 \mathrm{mmol}, 1 \mathrm{eq})$, DMAP $(.036 \mathrm{gm}, 0.5 \mathrm{mmol}, 0.31 \mathrm{eq})$ and triethylamine $(0.62 \mathrm{gm} .6 .2 \mathrm{mmol} . \mathrm{l} \mathrm{eq})$ at $0^{\circ} \mathrm{C}$ and stimed for 24 hrs. Alter that solvent was removed in valcum. the residue laken in ethyl 
(C) 2002 American Chemical Society, J. Org. Chem., Rajesh jo010981d Supporting Info Page 10 'acetate. washed with saturated seritium bicarbonate solution $(3 \times 10 \mathrm{~mL}), i 0 \% \mathrm{HCl}$ solution $(1 \times 10 \mathrm{~mL})$ and finally with water. The combined organic layers were dried over sodium sulfate and concentration of solvent resulted $\mathbb{N}$-cinnamoylated product $\mathbf{5 a}$, which was directly used for the next step. 5a: 'H NMR (400 MHz, $\left.\mathrm{CDCl}_{3}\right) \delta 7.73(\mathrm{~s}, 1 \mathrm{H}), 7.67$ $(\mathrm{d}, J=15.3 \mathrm{~Hz}, 1 \mathrm{H}), 7.36-7.31(\mathrm{~m}, 4 \mathrm{H}), 7.27-7.21(\mathrm{~m}, 6 \mathrm{H}) .6 .89(\mathrm{~d}, J=9 \mathrm{~Hz}, 4 \mathrm{H}), 6.80$ (d. $J=9 \mathrm{~Hz}, 2 \mathrm{H}), 6.24(\mathrm{~d}, J=15.3 \mathrm{~Hz}, 1 \mathrm{H}), 5.02(\mathrm{~s}, 2 \mathrm{H}), 3.81(\mathrm{~s}, 3 \mathrm{H}), 3.70(\mathrm{~s}, 3 \mathrm{H})$; IR $\left(\mathrm{CH}_{2} \mathrm{Cl}_{2}\right): 3425,2997,2835,1710.1510,1249 \mathrm{~cm}^{-1}$

To the $\mathrm{N}$-cinnamoylated dehydro amino ester in $\mathrm{MeOH}$ and $\mathrm{H}_{2} \mathrm{O}(4: 1)$ solution $(2.5 \mathrm{~mL}$ /mmol) was added $1.5 \mathrm{mmol}$ of $\mathrm{LiOH} . \mathrm{H}_{2} \mathrm{O}$ and the reaction mixture was stirred for 4 hours at rt. Then solvent was removed, washed with ether $(2 \times 10 \mathrm{~mL})$, neutralized with IN HCl ( $\mathrm{pH} 2$ ). The solid acid was filtered, dried and used for the next coupling. To a stirred solution of $\mathrm{N}$-cinnamoylated dehydro aminoacid $(1.65 \mathrm{~g}, 4 \mathrm{mmol}, 1 \mathrm{eq})$ in $20 \mathrm{~mL}$ THF-DMSO mixture at $-10^{\circ} \mathrm{C}$ was added $0.56 \mathrm{~mL}$ of triethylamine $(0.404 \mathrm{~g}, 4 \mathrm{mmol}, 1$ eq) and $0.31 \mathrm{~mL}$ of methyl chloroformate (0.378 gim, $4 \mathrm{mmol}, \mathrm{l} \mathrm{eq})$ and stirred for 2 minutes, followed by addition of Methyl-L-Leucinate (0.87g, $4.8 \mathrm{mmol}, 1.2 \mathrm{eq})$ and triethylamine (1.12 mL. $8 \mathrm{mmol} .2 .4 \mathrm{eq})$ and the reaction mixture was stirred for 3 to 4 hours. After that solvent was removed completely and taken in ethyl acetate. washed with saturated citric acid solution $(1 \times 10 \mathrm{~mL})$, sodium bicarbonate solution $(3 \times 10 \mathrm{~mL})$ and brine. The organic layer was dried over anhydrous sodium sulfate and concentration of the solvent and finally column chromatography yielded $6 \mathrm{a}$ in 55\% yield. 6a: 'H NMR (400 $\left.\mathrm{MHz}, \mathrm{CDCl}_{3}\right) \& 8.45(1 \mathrm{H}, \mathrm{d} . J=7.08 \mathrm{~Hz}) ; 7.85(1 \mathrm{H}, \mathrm{s}): 7.603(1 \mathrm{H}, \mathrm{d}, . J=15.4 \mathrm{~Hz})$; $7.16-7.06(10 \mathrm{H}, \mathrm{m}) ; 6.68(2 \mathrm{H} . \mathrm{d}, J=8.08 \mathrm{~Hz}) ; 6.58(2 \mathrm{H} . \mathrm{d} . .1 \div 5.88 \mathrm{~Hz}): 0.19(1 \mathrm{H}, \mathrm{d}, J$ $=15.3 \mathrm{~Hz}): 4.95(1 \mathrm{H} . \mathrm{d} . J=14.88 \mathrm{~Hz}): 4.83(1 \mathrm{H} . \mathrm{d} . J=15.36 \mathrm{~Hz}, 10 \mathrm{~Hz}): 3.68(3 \mathrm{H.s})$; 
(C) 2002 American Chemical Society, J. Org. Chem., Rajesh jo010981d Supporting Info Page 11

$3.58(3 \mathrm{H}, \mathrm{s}) ; 1.76 \sim 1.38(2 \mathrm{H} . \mathrm{m}) ; 1.17 \sim 1.11(1 \mathrm{H}, \mathrm{m}) ; 0.902(3 \mathrm{H}, \mathrm{d}, J=5.6 \mathrm{~Hz}) ; 0.79$

$(3 \mathrm{H}$. d. $J=6.56 \mathrm{~Hz}) ;$ FTIR $\left(\mathrm{CH}_{2} \mathrm{Cl}_{2}\right): 3298,3059,2956.2870,1744,1647,1611,1546$

$\mathrm{cm}^{-1}$; Anal. Calcd. for $\mathrm{C}_{33} \mathrm{H}_{36} \mathrm{O}_{5} \mathrm{~N}_{2}: \mathrm{H} 6.71 \mathrm{C} 73.31 \mathrm{~N} 5.18 \mathrm{O} 14.8$ Found $\mathrm{H} 6.5 \mathrm{C} 73.52$ N 5.35.O 14.5.

Coupling of (L)-leucine methyl ester with N-Cinnamoylated-dehydro-aminoacid (6b): Yield, 61\%; ${ }^{1} \mathrm{H}$ NMR $\left(400 \mathrm{MHz}, \mathrm{CDCl}_{3}\right) \delta 8.46(1 \mathrm{H} . \mathrm{d} . J=2.08 \mathrm{~Hz}) ; 7.93(1 \mathrm{H}, \mathrm{s})$; $7.72(1 \mathrm{H}, \mathrm{d}, J=9.04 \mathrm{~Hz}) ; 7.33 \sim 6.99(15 \mathrm{H}, \mathrm{m}) ; 6.62(1 \mathrm{H}, \mathrm{d}, J==7.32 \mathrm{~Hz}) ; 5.08-4.93(1 \mathrm{H}$, $\mathrm{m}) ; 3.72(3 \mathrm{H}, \mathrm{s}) ; 3.66(2 \mathrm{H}, \mathrm{s}) ; 1.83 \sim 1.67(1 \mathrm{H}, \mathrm{m}) ; 0.94(\mathrm{~d}, 3 \mathrm{H}, J=5.6 \mathrm{~Hz}), 0.93(3 \mathrm{H}, \mathrm{d}, J$ $=5.6 \mathrm{~Hz})$. FTIR $\left(\mathrm{CH}_{2} \mathrm{Cl}_{2}\right): 3398,3058,2927,2855,1718,1598,1505 \mathrm{~cm}^{-1}$.

Coupling of (L)-aspartate methyl ester with $\mathrm{N}$-Cinnamoylated-dehydro-aminoacid (6c): Yield, 59\%; ' ${ }^{H}$ NMR $\left(400 \mathrm{MHz}, \mathrm{CDCl}_{3}\right) \delta 8.69(1 \mathrm{H}, \mathrm{s}), 7.73(1 \mathrm{H}, \mathrm{s}), 7.68(1 \mathrm{H}, \mathrm{d}, J$ $=15.2 \mathrm{~Hz}) ; 7.61(1 \mathrm{H}, \mathrm{d}, J=8.56 \mathrm{~Hz}) ; 7.72(1 \mathrm{H}, \mathrm{d}, J=9.04 \mathrm{~Hz}) ; 7.33 \sim 7.19(12 \mathrm{H}, \mathrm{m}) ;$ $6.97-6.81(4 \mathrm{H}, \mathrm{m}) ; 6.26(1 \mathrm{H}, \mathrm{d}, J=15.6 \mathrm{~Hz}) ; 3.92-3.82(1 \mathrm{H}, \mathrm{m}) ; 3.74(3 \mathrm{H}, \mathrm{s}) ; 3.69(3 \mathrm{H}$, s); 3.68 (3H.s).

Cobalt (II) chloride catalysed epoxide Opening by the dehydro amine $2 b(8 a$ \& 8 b): To a stirred solution of cobalt (11) chloride $(10 \mathrm{~mol} \%)$ in acetonitrile $(15 \mathrm{ml}$.) was added 7a (222 $\mathrm{mg}, 0.66 \mathrm{mmol}, 1 \mathrm{eq})$ and $3 \mathbf{b}(196 \mathrm{mg}, 0.66 \mathrm{mmol}, \mathrm{leq})$ and the mixture stirred at ambient temperature under nitrogen atmosphere, for $10-12$ hours. The solvent is evaporated under reduced pressure and the residue was taken in ethylacetate and washed thoroughly with saturated solution of sodium bicarbonate solution and water. Drying (sodium sulphate) and evaporation of solvent gave a residue. which was subjected to column chromatography over silicagel (10\% EIOAc / hexanc) to afford 8 a as a gum in $45-50 \%$ yield. $8 \mathrm{a}$ was observed as a mixture of very minor sym diastereomer (higher $\mathrm{R}_{\mathrm{f}}$ 
(C) 2002 American Chemical Society, J. Org. Chem., Rajesh jo010981d Supporting Info Page 12 value in TLC) and major anti diastereomer (lower $\mathrm{R}_{f}$ value in TLC). Only the anti diastereomer was isolated and the data for it has been presented here. ${ }^{1} \mathrm{H}$ NMR $\left(400 \mathrm{MHz}, \mathrm{CDCl}_{3}\right)(E)-8 \mathrm{a}: \delta 7.53(\mathrm{~m}, 1 \mathrm{H}), 7.46(\mathrm{~s}, 1 \mathrm{H}), 7.12-7.34(\mathrm{~m}, 8 \mathrm{H}), 6.94(\mathrm{~m}, 1 \mathrm{H})$ $6.83(\mathrm{~m}, 2 \mathrm{H}), 6.60(\mathrm{~m}, 2 \mathrm{H}), 5.07(\mathrm{~m}, 1 \mathrm{H}), 4.70(\mathrm{bs}, 1 \mathrm{H}), 4.42(\mathrm{~m}, 1 \mathrm{H}), 4.35(\mathrm{bs}, 1 \mathrm{H})$, $4.04(\mathrm{~d}, J=12.6 \mathrm{~Hz}, 1 \mathrm{H}), 3.98(\mathrm{~d}, J=12.6 \mathrm{~Hz}, 1 \mathrm{H}), 3.87(\mathrm{~m}, 1 \mathrm{H}), 3.73(\mathrm{~s}, 3 \mathrm{H}), 3.69(\mathrm{~s}$, 3H), $3.63(\mathrm{~s}, 3 \mathrm{H}), 3.37(\mathrm{~m}, 1 \mathrm{H}), 2.21(\mathrm{~m}, 1 \mathrm{H}), 2.07(\mathrm{~m}, 1 \mathrm{H}), 1.95(\mathrm{~s}, 3 \mathrm{H}) ;$ Mass $(\mathrm{m} / \mathrm{z})$ : $631(\mathrm{M}+1,20 \%), 386(100 \%), 297(20 \%), 212(50 \%), 175(15 \%), 126(20 \%), 91(20 \%)$; IR $\left(\mathrm{CCl}_{4}\right): 3420,3040,3015,2940,1725,1700,1590 \mathrm{~cm}^{-1} ;[\alpha]_{D}=-36.36(\mathrm{c}=0.008$, $\mathrm{CH}_{2} \mathrm{Cl}_{2}$ ).

Similarly, to a stirred solution of cobalt (II) chloride (10 mol\%) in acetonitrile (15 mL) was added $7 \mathbf{b}(500 \mathrm{mg}, 1.7 \mathrm{mmol}, 1 \mathrm{eq})$ and $\mathbf{3 b}(504 \mathrm{mg}, 0.66 \mathrm{mmol}, 1 \mathrm{eq})$ and the reaction mixture stirred at ambient temperature under nitrogen atmosphere for 15-17 hours. The solvent is evaporated under reduced pressure and the residue was taken in ethyl acetate and washed thoroughly with saturated solution of sodium bicarbonate and water. Drying (sodium sulphate) and evaporation of solvent gave a residue which was column chromatographed over silica gel (10\% EtOAc : hexanes) to afford $\mathbf{8 b}$ as a gum in $45-50 \%$ yield. 8a was observed as a mixture of very minor syn diastereomer (higher $R_{f}$ value in TLC) and major anti diastereomer (lower $\mathrm{R}_{\mathrm{f}}$ value in TLC). Only the anti diastereomer was isolated and the data for it has been presented here. ${ }^{1} \mathrm{H}$ NMR $\left(400 \mathrm{MHz}, \mathrm{CDCl}_{3}\right)(\boldsymbol{E})-8 \mathbf{b}: \delta 7.55(1 \mathrm{H}, \mathrm{s}), 7.28 \sim 6.92 \cdot(8 \mathrm{H}, \mathrm{m}), 6.81(2 \mathrm{H}, \mathrm{m}), 6.74(2 \mathrm{H}, \mathrm{d}$, $J=8.8 \mathrm{~Hz}), 6.50(2 \mathrm{H}, \mathrm{d}, J=9.04 \mathrm{~Hz}), 4.75(1 \mathrm{H}, \mathrm{d}, J=3.68 \mathrm{~Hz}), 4.59(1 \mathrm{H}, \mathrm{d}, J=3.64 \mathrm{~Hz})$, $4.31(1 \mathrm{H}, \mathrm{dt}, J=5.12 \mathrm{~Hz}, 9.28 \mathrm{~Hz}), 4.01(1 \mathrm{H}, \mathrm{d}, J=13.2 \mathrm{~Hz}), 3.87(1 \mathrm{H}, \mathrm{d}, J=12.96 \mathrm{~Hz})$, $3.84(3 \mathrm{H}, \mathrm{s}), 3.65(3 \mathrm{H}, \mathrm{s}), 3.641(3 \mathrm{H}, \mathrm{s}), 1.28 \sim 1.08(2 \mathrm{H}, \mathrm{m}), 0.97 \sim 0.76(1 \mathrm{H}, \mathrm{m}), 0.68$ 
$(3 \mathrm{H}, \mathrm{d}, J=6.56 \mathrm{~Hz}), 0.59(3 \mathrm{H}, \mathrm{d}, J=6.36 \mathrm{~Hz})$, Mass $(\mathrm{m} / \mathrm{z}): 589(\mathrm{M}+1,50 \%), 386$ (100\%), 297, 212; FTIR $\left(\mathrm{CH}_{2} \mathrm{Cl}_{2}\right): 3404,2956,1743,1697,1676,1509,1438 \mathrm{~cm}^{-1} ;[\alpha]_{D}$ $=+16\left(\mathrm{c}=0.042, \mathrm{CH}_{2} \mathrm{Cl}_{2}\right)$.

Titanium tetraisopropoxide mediated transesterification of $8 \mathrm{~b}(8 \mathrm{c})$ : To the Compound $8 \mathrm{~b}$ (352 mg, $0.6 \mathrm{mmol}, \mathrm{l} \mathrm{eq})$ in allyl alcohol $(15 \mathrm{~mL})$ was added Ti $\left(\mathrm{OPr}^{\mathrm{i}}\right)_{4}$ ( $85 \mathrm{mg}, 0.3 \mathrm{mmol}, 0.5 \mathrm{eq}$ ) and the resulting mixture was refluxed at $100^{\circ} \mathrm{C}$ for 16 hours. Removal of solvent under reduced pressure gave a residue, which was taken in ethyl acetate, and the murky solution was passed through a celite pad. Removal of solvent gave a residue which was chromatographed over silicagel (EtOAc / hexane) to afford $8 \mathrm{c}$ (55$60 \%)$ as a gum. ' $\mathrm{H}$ NMR $\left(400 \mathrm{MHz}, \mathrm{CDCl}_{3}\right) 8 \mathrm{c}: \delta 7.50(\mathrm{~s}, 1 \mathrm{H}), 7.21-7.00(\mathrm{~m}, 10 \mathrm{H}), 6.88$ $(\mathrm{d}, J=8.8 \mathrm{~Hz}, 1 \mathrm{H}), 6.61(\mathrm{~d}, J=8.8 \mathrm{~Hz}, 2 \mathrm{H}), 6.41(\mathrm{~d}, J=8.8 \mathrm{~Hz}, 2 \mathrm{H}), 5.92(\mathrm{~m}, 1 \mathrm{H}), 5.78$ $(\mathrm{m}, 1 \mathrm{H}), 5.33(\mathrm{~d}, J=13.44 \mathrm{~Hz}, 1 \mathrm{H}), 5.19(\mathrm{~m}, 2 \mathrm{H}), 5.11(\mathrm{~d}, J=10.48 \mathrm{~Hz}, 1 \mathrm{H}), 4.86(\mathrm{~d}, J$ $=6.12 \mathrm{~Hz}, 1 \mathrm{H}), 4.66(\mathrm{~d}, J=3.92 \mathrm{~Hz}, 1 \mathrm{H}), 4.51(\mathrm{~d}, J=3.44 \mathrm{~Hz}, 1 \mathrm{H}), 4.46(\mathrm{~d}, J=5.64$ $\mathrm{Hz}, 2 \mathrm{H}), 4.25(\mathrm{dt}, J=9$ and $5.12 \mathrm{~Hz}, 1 \mathrm{H}), 3.93(\mathrm{~d}, J=13 \mathrm{~Hz}, 1 \mathrm{H}), 3.80(\mathrm{~d}, J=13$ $\mathrm{Hz}, 1 \mathrm{H}), 3.55(\mathrm{~s}, 3 \mathrm{H}), 1.50(\mathrm{~m}, 1 \mathrm{H}), 0.79(\mathrm{~m}, 2 \mathrm{H}), 0.60(\mathrm{~d}, J=6.56 \mathrm{~Hz}, 3 \mathrm{H}), 0.52(\mathrm{~d}, J$ $=6.32 \mathrm{~Hz}, 3 \mathrm{H})$. Mass $(\mathrm{m} / \mathrm{z}): 641(\mathrm{M}+1,80 \%), 412(100 \%), 323(10 \%), 212(30 \%)$. FTIR $\left(\mathrm{cm}^{-1}\right) 3404$ (broad), 2957, 1741, 1679, 1509, $1244 \mathrm{~cm}^{-1} ;[\alpha]_{\mathrm{D}}=+3.2(\mathrm{c}=0.005$, $\mathrm{CH}_{2} \mathrm{Cl}_{2}$ ).

Ring closing Metathesis of $8 \mathrm{c}(9)$ : To a stirring solution of ruthenium methylidene catalyst (Grubbs catalyst) $(15.4 \mathrm{mg}, 0.0187 \mathrm{mmol}, 0.15 \mathrm{eq})$ in dichloromethane $(0.6 \mathrm{mM}$ solution) under nitrogen, the diene $8 \mathrm{c}(80 \mathrm{mg}, 0.125 \mathrm{mmol}$. leq ) was added and the mixture was refluxed for $10-12$ hours. At this stage an additional ruthenium methylidine catalyst (10 mol\%) was added and the mixture was further refluxed for 8 hours. The 
(C) 2002 American Chemical Society, J. Org. Chem., Rajesh jo010981d Supporting Info Page 14 solvent was evaporated to yield a residue which was chromatographed over silica gel (30\% EtOAc : hexane ) to afford the Major isomer trans- 9 (40-45\%) as a gum. trans(9): ' ${ }^{\mathrm{H}} \mathrm{NMR}\left(400 \mathrm{MHz}, \mathrm{CDCl}_{3}\right) \delta 8.21(\mathrm{bs}, 1 \mathrm{H}), 7.60(\mathrm{~s}, 1 \mathrm{H}), \quad 7.10-7.42(\mathrm{~m}, 7 \mathrm{H})$, 6.86-6.93 (m, 3H), $6.57(\mathrm{~d}, J=9.04 \mathrm{~Hz}, 2 \mathrm{H}), 6.49(\mathrm{~d}, J=9.04 \mathrm{~Hz}, 2 \mathrm{H}), 5.91(\mathrm{dt}, J=$ 16.72 and $5.84 \mathrm{~Hz}, 1 \mathrm{H}), 5.34$ (ddd, $J=17.3,3$ and $1.6 \mathrm{~Hz}, 1 \mathrm{H}), 4.67-4.82(\mathrm{~m}, 2 \mathrm{H})$, $4.63(\mathrm{bs}, 2 \mathrm{H}), 4.69(\mathrm{bs}, 2 \mathrm{H}), 4.50(\mathrm{dd}, J=4.88$ and $12.2 \mathrm{~Hz}, 1 \mathrm{H}), 4.05(\mathrm{~d}, J=13.92$ $\mathrm{Hz}, 1 \mathrm{H}), 3.98(\mathrm{~d}, J=13.92 \mathrm{~Hz}, 1 \mathrm{H}), 3.71(\mathrm{~s}, 3 \mathrm{H}), 1.86(\mathrm{~m}, 1 \mathrm{H}), 1.55-1.71(\mathrm{~m}, 2 \mathrm{H})$, 0.95 (d, $J=6.36 \mathrm{~Hz}, 3 \mathrm{H}), 0.91(\mathrm{~d}, J=6.36 \mathrm{~Hz}, 3 \mathrm{H})$. FT-IR $\left(\mathrm{CH}_{2} \mathrm{Cl}_{2}\right): 3360,2257$, $2925,1739,1659,1511,1453 \mathrm{~cm}^{-1} ;$ Mass $(\mathrm{m} / \mathrm{z}): 613\left(\mathrm{M}^{+}, 100 \%\right), 555(25 \%), 412$ $(30 \%), 212(50 \%) ;[\alpha]_{\mathrm{D}}=-3\left(\mathrm{c}=0.005, \mathrm{CH}_{2} \mathrm{Cl}_{2 .}\right) ;(11):{ }^{\prime} \mathrm{H} \mathrm{NMR}\left(400 \mathrm{MHz}, \mathrm{CDCl}_{3}\right)$ $\delta 7.19-7.52(\mathrm{~m}, 7 \mathrm{H}), 6.91-6.97(\mathrm{~m}, 3 \mathrm{H}), 6.61(\mathrm{~d}, J=9.04 \mathrm{~Hz}, 2 \mathrm{H}), 6.51(\mathrm{~d}, J=9.04$ $\mathrm{Hz}, 2 \mathrm{H}), 5.96(\mathrm{dt}, J=16.69$ and $5.81 \mathrm{~Hz}, 1 \mathrm{H}), 5.39$ (ddd, $J=17,3.1$ and $1.8 \mathrm{~Hz}, 1 \mathrm{H}$ ), $4.72-4.88(\mathrm{~m}, 2 \mathrm{H}), 4.71(\mathrm{bs}, 2 \mathrm{H}), \quad 4.53(\mathrm{dd}, J=4.8$ and $12 \mathrm{~Hz}, 1 \mathrm{H}), 4.01(\mathrm{~d}, J=14.1$ $\mathrm{Hz}, 1 \mathrm{H}), 3.88(\mathrm{~d}, J=14.1 \mathrm{~Hz}, 1 \mathrm{H}), 3.69(\mathrm{~s}, 3 \mathrm{H}), 3.13(\mathrm{~m}, 2 \mathrm{H}), 2.94(\mathrm{~m}, 1 \mathrm{H}), 2.87$ (ddd, $J=16.7,4.1$ and $2.1 \mathrm{~Hz}, 1 \mathrm{H}), 2.76(\mathrm{ddd}, J=16.7,4.2$ and $4.3 \mathrm{~Hz}, 1 \mathrm{H}), 1.86(\mathrm{~m}, 1 \mathrm{H})$, $1.551 .71(\mathrm{~m}, 2 \mathrm{H}), 0.95(\mathrm{~d}, J=6.36 \mathrm{~Hz}, 3 \mathrm{H}), 0.91(\mathrm{~d}, J=6.36 \mathrm{~Hz}, 3 \mathrm{H})$. Mass $(\mathrm{m} / \mathrm{z})$ : $615\left(\mathrm{M}^{+}, 100 \%\right), 557(35 \%), 317(25 \%), 214(37 \%)$. 